\title{
Antibody-Drug Conjugates Using Mouse-Canine Chimeric Anti-Dog Podoplanin Antibody Exerts Antitumor Activity in a Mouse Xenograft Model
}

\author{
Yukinari Kato, ${ }^{1,2}$ Yuji Ito, ${ }^{3}$ Tomokazu Ohishi, ${ }^{4}$ Manabu Kawada, ${ }^{4}$ Takuro Nakamura, \\ Yusuke Sayama, Masato Sano, ${ }^{1}$ Teizo Asano, Miyuki Yanaka, ${ }^{1}$ Saki Okamoto, \\ Saori Handa, ${ }^{1}$ Yu Komatsu, Junko Takei, ${ }^{1}$ and Mika K. Kaneko ${ }^{1}$
}

Antibody-drug conjugates (ADCs), which consist of a monoclonal antibody (mAb), a linker, and a payload, can deliver a drug to cancer tissues. We previously produced an anti-dog podoplanin (dPDPN) mAb, PMab-38, which reacts with dPDPN-expressing canine melanomas and squamous cell carcinomas (SCCs), but not with dPDPN-expressing canine type I alveolar cells or lymphatic endothelial cells, indicating that PMab-38 possesses cancer specificity. In this study, we developed an ADC, P38B-DM1, using the mouse-canine chimeric anti-dPDPN antibody, P38B as the antibody, a peptide linker, and emtansine as the payload using the chemical conjugation by affinity peptide (CCAP) method. We investigated its cytotoxicity against dPDPN-overexpressed Chinese hamster ovary (CHO/dPDPN) cells in vitro and its antitumor activity using a mouse xenograft model of CHO/dPDPN cells. P38B-DM1 showed cytotoxicity to $\mathrm{CHO} / \mathrm{dPDPN}$ cells in a dose-dependent manner in vitro. Furthermore, P38B-DM1 exhibited higher antitumor activity than P38B in the mouse xenograft model. These results suggest that P38B-DM1, developed using the CCAP method, is useful for antibody therapy against dPDPN-expressing canine SCCs and melanomas.

Keywords: antibody-drug conjugate, dog podoplanin, monoclonal antibody

\section{Introduction}

A NTIBODY-DRUG CONJUGATES (ADCs), which consist of an antibody, a linker, and a payload, possess the ability to deliver a drug to specific cells. Several sitespecific chemical conjugation strategies with native monoclonal antibodies (mAbs) have been developed, including residue-selective labeling, disulfide rebridging, and affinitypeptide-mediated site-specific chemical conjugation technologies. ${ }^{(1)}$ The most popular antibody conjugation system is an amine coupling, using the activated carboxylate moiety, which has been used to generate a typical ADC, T-DM1 (Kadcyla), used against breast cancers. However, to overcome difficulties involved in controlling the modification site and the drug concentration (drug-to-antibody ratio [DAR]), site-specific modification of the antibody has been favored. ${ }^{(2)}$ Recently, chemical conjugation by affinity peptide (CCAP) has been developed using affinity peptide to the antibody $\mathrm{Fc}$ fragment as a novel site-specific conjugation system. ${ }^{(3)}$ Utilizing CCAP, various kinds of therapeutic molecules, from low molecular weight compounds to proteins, can be attached to the antibody Fc through the affinity peptide. In this study, we investigated an ADC consisting of a conjugate between antibody and human serum albumin (HSA)-modified with emtansine (DM1; a tubulin polymerization inhibitor).

Podoplanin (PDPN), which is also known as Aggrus, T1alpha, PA2.26, OTS-8, and gp36, has been reported to be expressed in normal tissues, including podocytes of the kidney, lymphatic endothelial cells, and type I alveolar cells of the lung. ${ }^{(4)}$ PDPN is also overexpressed in many cancers, such as glioblastomas, ${ }^{(5)}$ lung cancers, ${ }^{(6)}$ and mesotheliomas. ${ }^{(7,8)}$ Several clinical studies have investigated the association between PDPN overexpression and poor disease prognosis, ${ }^{(9)}$ suggesting that anti-PDPN mAbs could be used for novel therapeutic strategies against cancer development and metastatic progression. ${ }^{(10)}$

\footnotetext{
${ }^{1}$ Department of Antibody Drug Development, Tohoku University Graduate School of Medicine, Sendai, Japan.

${ }^{2}$ New Industry Creation Hatchery Center, Tohoku University, Sendai, Japan.

${ }^{3}$ Department of Chemistry and Bioscience, Graduate School of Science and Engineering, Kagoshima University, Kagoshima, Japan.

${ }^{4}$ Institute of Microbial Chemistry (BIKAKEN), Numazu, Microbial Chemistry Research Foundation, Shizuoka, Japan.
}

(c) Yukinari Kato et al. 2020; Published by Mary Ann Liebert, Inc. This Open Access article is distributed under the terms of the Creative Commons License (http://creativecommons.org/licenses/by/4.0), which permits unrestricted use, distribution, and reproduction in any medium, provided the original work is properly cited. 
Previously, we established an anti-dog podoplanin (dPDPN), PMab-38 mAb. ${ }^{(11)}$ PMab-38 did not react with lymphatic endothelial cells, and recognized dPDPN of renal epithelial cells weakly ${ }^{(11)}$ although dPDPN is expressed in those cells. ${ }^{(12)}$ Immunohistochemical analyses demonstrated that PMab-38 stained canine squamous cell carcinomas ${ }^{(13)}$ and canine melanomas, ${ }^{(14)}$ indicating that PMab-38 demonstrated cancer specificity. Epitope mapping studies revealed Tyr67 and Glu68 of dPDPN to be the critical epitopes of PMab-38. ${ }^{(15)}$ Furthermore, we produced a mouse-canine chimeric antidPDPN antibody, P38B, derived from PMab-38. ${ }^{(16)}$ P38B possesses antibody-dependent cellular cytotoxicity (ADCC) and complement-dependent cytotoxicity (CDC) activities against Chinese hamster ovary $(\mathrm{CHO}) / \mathrm{dPDPN}$ cells. Although $\mathrm{P} 38 \mathrm{~B}$ exhibited antitumor activity in mouse xenograft models using $\mathrm{CHO} / \mathrm{dPDPN},{ }^{(17)}$ that activity was insufficient for targeting canine cancers. In this study, we investigated whether an ADC of P38B (P38B-DM1) showed superior antitumor activity in a $\mathrm{CHO} / \mathrm{dPDPN}$ xenograft model.

\section{Materials and Methods}

\section{Antibodies}

PMab-38, a mouse anti-dPDPN mAb was developed in our previous study. ${ }^{(1)}$ To develop the mouse-canine chimeric antibody $\mathrm{P} 38 \mathrm{~B}, \mathrm{~V}_{\mathrm{H}}$ of PMab-38 and $\mathrm{C}_{\mathrm{H}}$ of canine immunoglobulin $\mathrm{G}(\mathrm{IgG})$ subclass B were subcloned into the pCAG-Ble vector (FUJIFILM Wako Pure Chemical Corporation, Osaka, Japan), and $\mathrm{V}_{\mathrm{L}}$ of PMab-38 and $\mathrm{C}_{\mathrm{L}}$ of canine IgG were subcloned into the pCAG-Neo vector (FUJIFILM Wako Pure Chemical Corporation). ${ }^{(16)}$ Using the ExpiFectamine $\mathrm{CHO}$ Transfection kit (Thermo Fisher Scientific, Inc., Waltham, MA), the expression vectors were transfected into ExpiCHO-S cells to express P38B antibody. P38B was purified using Protein G-Sepharose (GE Healthcare Biosciences, Pittsburgh, PA).

\section{Preparation of $A D C P 38 B-D M 1$}

For the preparation of the ADC using P38B, azide-modified CCAP peptide (FNMQQQ(Lys[Azide])RFYEALHDPNLNE EQRNARI(Lys[Azide])SIRDDPSRR(Lys[Azide])RR(Lys [Azide])RR(Lys[Azide])-CONH2), where Lys[Azide] is 5-azide-2-amino pentanoic acid) derived from Z34C peptide $^{(18)}$ was used. This CCAP peptide was N-terminally modified with disuccinimidyl glutarate to give the CCAP reagent ( $N$-terminal succinimidyl glutaryl peptide). P38B $(4.5 \mathrm{~mL}$, $5.1 \mathrm{mg} / \mathrm{mL} ; 34 \mu \mathrm{M}$ ) in phosphate-buffered saline (PBS) was mixed with $22 \mu \mathrm{L}$ of $27.9 \mathrm{mM}$ CCAP reagent dissolved in dimethyl sulfoxide and incubated for 1 hour at room temperature.

The reaction of antibody with CCAP peptide generated the monovalent and divalent antibodies owing to the two modified sites of CCAP peptide on Fc. To remove the unmodified and the divalent modified antibodies, the reaction mixture was subjected to affinity chromatography conjugated with Z33 affinity peptide (Acetyl-FNMQQQRRFYEALHDPNL NEEQRNARIRSIRDDPSGSGSK-NH2). The monovalent modified antibody was recovered by eluting with mild acidic buffer. Based on ion-exchange chromatography, the purity of monovalent modified antibody was estimated to be $95 \%$, leaving the residual contaminant of $5 \%$ unmodified antibody.

The modification of HSA with DM-1 was performed as follows. HSA $(20 \mathrm{mg})$ dissolved in $1.35 \mathrm{~mL}$ of $50 \mathrm{mM}$ HEPES
(pH 7.0) was reduced with $0.5 \mathrm{mM}$ dithiothreitol (DTT). The partially reduced HSA was supplied to a Hi Trap Capto Q column (GE Healthcare Biosciences) equilibrated with $50 \mathrm{mM}$ HEPES buffer ( $\mathrm{pH} 7.0$ ), washed with the same buffer, and eluted with buffer containing $1 \mathrm{M} \mathrm{NaCl}$. The eluted HSA $(50 \mu \mathrm{M})$ was reacted with $250 \mu \mathrm{M}$ dibenzocyclooctyne (DBCO)-PEG4-Maleimide (Click Chemistry Tools) and incubated for 2 hours at a room temperature. The DBCOmodified HSA formed $(7 \mu \mathrm{M})$ was subsequently reacted with a 20-fold excess of DM1-SMCC (MedChemExpress) for 12 hours at $37^{\circ} \mathrm{C}$.

After the reaction, the sample was supplied to a Hi Trap Capto Q column (GE Healthcare Biosciences) equilibrated with $50 \mathrm{mM}$ HEPES buffer ( $\mathrm{pH} 7.0$ ), washed with the same buffer, and eluted with buffer containing $1 \mathrm{M} \mathrm{NaCl}$. The drug (DM-1) attachment efficiency to the HSA (DAR) was estimated to be 2.9 using matrix-associated laser desorption/ionization time-of-flight mass spectroscopy (autoflex speed TOF; Bruker Daltonics).

Finally, azide-modified Z33-monovalent-modified antibody $(10 \mu \mathrm{M})$ and DM-1/DBCO-modified HSA $(20 \mu \mathrm{M})$ were reacted at $\mathrm{pH} 7.0$ for 12 hours at room temperature through the click chemistry. The generation of antibody-HSA conjugate was confirmed by sodium dodecyl sulfate-polyacrylamide gel electrophoresis (SDS-PAGE). The reaction mixture was subjected to a Z33 affinity peptide column to remove the excess HSA eluted in the excluded fraction. The bound HSA-monovalent modified antibody was eluted by $0.1 \mathrm{M}$ acetic acid buffer ( $\mathrm{pH} 4.0$ ) and dialyzed in PBS for use.

\section{Cell lines and animals}

We previously inserted dPDPN with an N-terminal PA tag (GVAMPGAEDDVV) ${ }^{(19)}$ and a C-terminal RAP tag (DMVNPGLEDRIE) $^{(20)}$-MAP tag (GDGMVPPGIEDK) ${ }^{(21)}$ (PA-dPDPN-RAP-MAP) in a pCAG-Ble vector (FUJIFILM Wako Pure Chemical Corporation). ${ }^{(1)}$ CHO-K1 cells (American Type Culture Collection, Manassas, VA) were transfected with pCAG-Ble/PA-dPDPN-RAP-MAP using Gene Pulser Xcell electroporation system (Bio-Rad Laboratories, Inc., Berkeley, CA) for developing CHO/dPDPN.

$\mathrm{CHO} / \mathrm{dPDPN}$ and $\mathrm{CHO}-\mathrm{K} 1$ cells were cultured using RPMI-1640 medium (Nacalai Tesque, Inc., Kyoto, Japan) supplemented with $10 \%$ heat-inactivated fetal bovine serum (Thermo Fisher Scientific, Inc.). Antibiotics, such as $100 \mathrm{U} / \mathrm{mL}$ penicillin, $100 \mu \mathrm{g} / \mathrm{mL}$ streptomycin, and $25 \mu \mathrm{g} / \mathrm{mL}$ amphotericin B (Nacalai Tesque, Inc.) were added into the medium. Cells were cultivated at $37^{\circ} \mathrm{C}$ in a humidified atmosphere of $5 \%$ $\mathrm{CO}_{2}$ and $95 \%$ air.

\section{Flow cytometry}

Using $0.25 \%$ trypsin $/ 1 \mathrm{mM}$ ethylenediaminetetraacetic acid (Nacalai Tesque, Inc.), CHO/dPDPN cells were harvested. Cells were treated with P38B $(0.01-10 \mu \mathrm{g} / \mathrm{mL})$, P38B-DM1 $(0.01-10 \mu \mathrm{g} / \mathrm{mL})$, or normal canine IgG $(10 \mu \mathrm{g} / \mathrm{mL}$; Jackson ImmunoResearch, Inc., PA) for 30 minutes at $4^{\circ} \mathrm{C}$, followed by treatment with fluorescein isothiocyanate-conjugated anticanine IgG (1:200; Thermo Fisher Scientific, Inc.). In all steps, the cells were washed with $1 \%$ bovine serum albumin in PBS. Fluorescence data were detected using the Cell Analyzer EC800 (Sony Corp., Tokyo, Japan). We repeated these experiments twice. 
Cytotoxicity assay

Cytotoxicity assay by cell viability assessment was conducted using Cell Cloning Kit-8 (CCK-8; Dojindo, Kumamoto, Japan). We prepared $96-$ well microplates by plating
$\mathrm{CHO} / \mathrm{dPDPN}$ and CHO-K1 at 6000 cells/200 $\mu \mathrm{L} /$ well. The plates were incubated for 24 hours at $37^{\circ} \mathrm{C}$ in the presence of $5 \% \mathrm{CO}_{2}$, then serially diluted antibodies were added in a volume of $20 \mu \mathrm{L}$ and cultured for $24-72$ hours. After that, the culture medium was replaced with $100 \mu \mathrm{L}$ of fresh medium

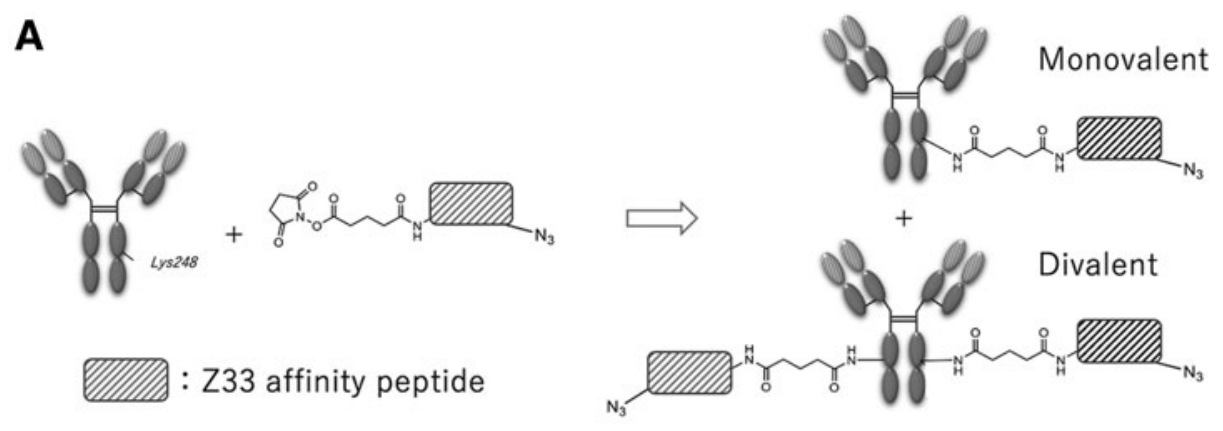

B
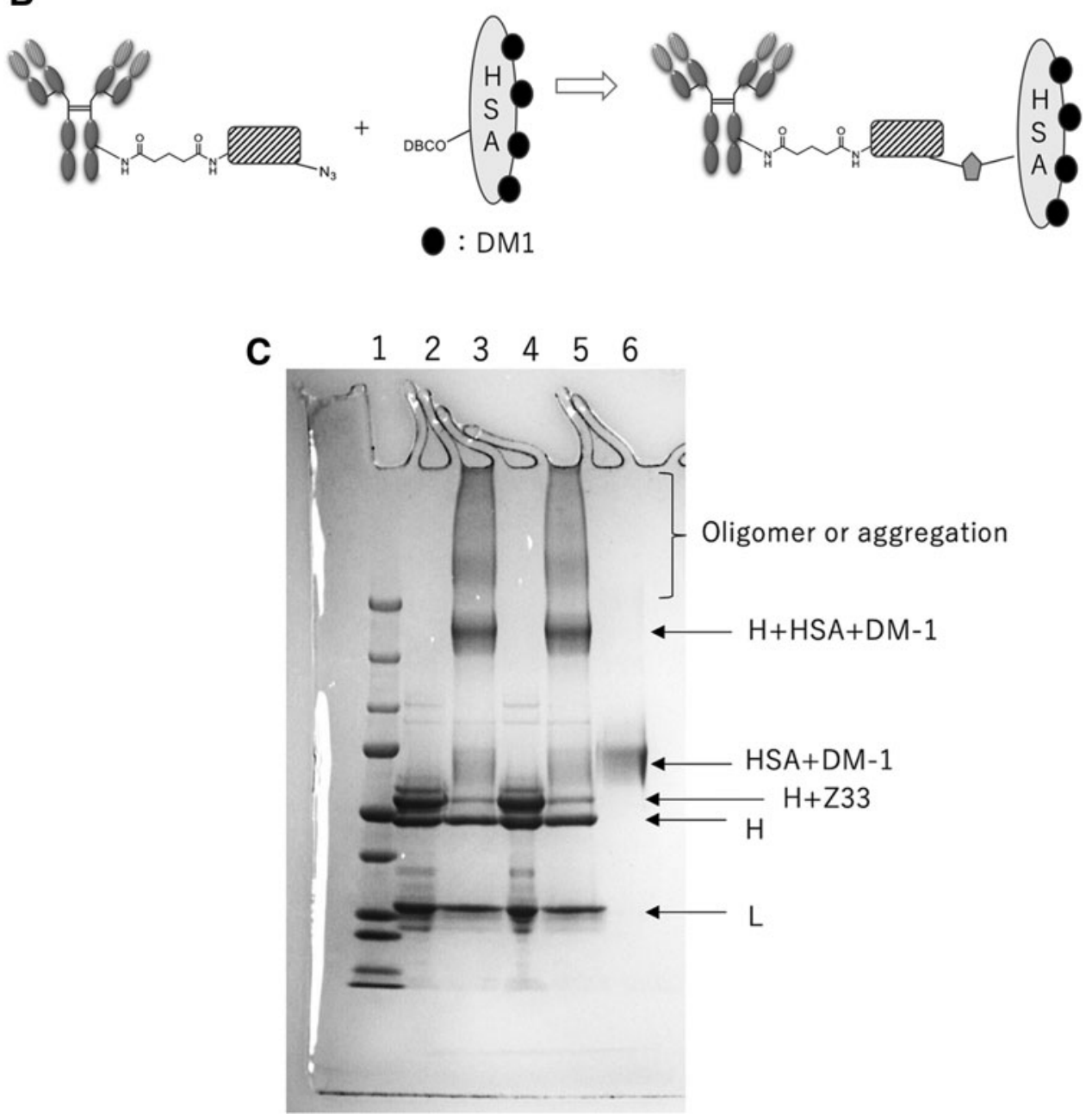

FIG. 1. Preparation of ADC P38B-DM1 using CCAP method. (A) The reaction of antibody with CCAP peptide generated the monovalent and divalent antibodies owing to the two modified sites of CCAP peptide on Fc. To remove the unmodified and the divalent-modified antibodies, the reaction mixture was subjected to to affinity chromatography conjugated with Z33 affinity peptide. (B) The modification of HSA with DM-1 was performed. (C) Sodium dodecyl sulfate-polyacrylamide gel electrophoresis of conjugate products under reducing conditions. Lane 1: molecular weight marker; Lane 2: Z33-azide monovalent modified P38B; Lane 3: Z33-azide monovalent modified P38B + DBCO-HSA-DM-1; Lane 4: Z33-azide monovalent modified P38Bf (core fucose-deficient version of P38B); Lane 5: Z33-azide monovalent-modified P38Bf + DBCO-HSA-DM-1; Lane 6: DBCO-HSA-DM-1. H: heavy chain, L: light chain. DBCO, dibenzocyclooctyne; ADC, antibody-drug conjugate; CCAP, chemical conjugation by affinity peptide; HSA, human serum albumin. 
with $10 \mu \mathrm{L} \mathrm{CCK}-8$ solution. After a further 5- to 6-hour incubation in a $\mathrm{CO}_{2}$ incubator, the absorbance at $450 \mathrm{~nm}$ was measured.

\section{Antitumor activity}

Female BALB/c nude mice ( 5 weeks old) were purchased from Charles River (Kanagawa, Japan) and used in experiments when they were 6 weeks old. All animal experiments were reviewed and approved by the Institutional Animal Care and Use Committee of Institute of Microbial Chemistry (Approval number: 2019-043) and were performed in accordance with relevant guidelines and regulations to minimize animal suffering.

CHO/dPDPN cells $\left(0.3 \mathrm{~mL}\right.$ at $1.33 \times 10^{8} / \mathrm{mL}$ in RPMI $)$ and $0.5 \mathrm{~mL}$ of BD Matrigel Matrix Growth Factor Reduced (BD Biosciences, San Jose, CA) were mixed, and $5 \times 10^{6}$ cells in $100 \mu \mathrm{L}$ were injected into the left flank of nude mice subcutaneously. One day after cell inoculation, $100 \mu \mathrm{g}$ of antibodies (P38B-DM1, P38B, or normal canine IgG) in $100 \mu \mathrm{L}$ of PBS were injected into the peritoneal cavity of each mouse. On days 8 and 14, additional $100 \mu \mathrm{g}$ of antibodies were in- jected into each mouse. As previously described, the tumor diameter and tumor volume were determined. ${ }^{(22)}$ The mice were killed 17 days after cell implantation.

All data were expressed as mean \pm standard error of mean. Statistical analysis was performed using the Tukey-Kramer test, with $p<0.05$ considered statistically significant.

\section{Results}

In this study, we developed an ADC, P38B-DM1, using the mouse-canine chimeric anti-dPDPN antibody, P38B as the antibody, a peptide linker, and emtansine as the payload using the CCAP method as given in Figure 1A and B. SDSPAGE of azide-modified Z33-monovalent modified antibody and its conjugate with DBCO-HSA-DM-1 (P38B-ADC) are given in Figure 1C.

Antibodies are affinity proteins and their ability to bind the target molecule with high sensitivity and specificity is crucial to their function. With this in mind, we examined the characteristics of P38B and P38B-DM1. Flow cytometry results (Fig. 2) showed that both P38B and P38B-DM1 exhibited similar reactions with $\mathrm{CHO} / \mathrm{dPDPN}$ cells in a dose-dependent
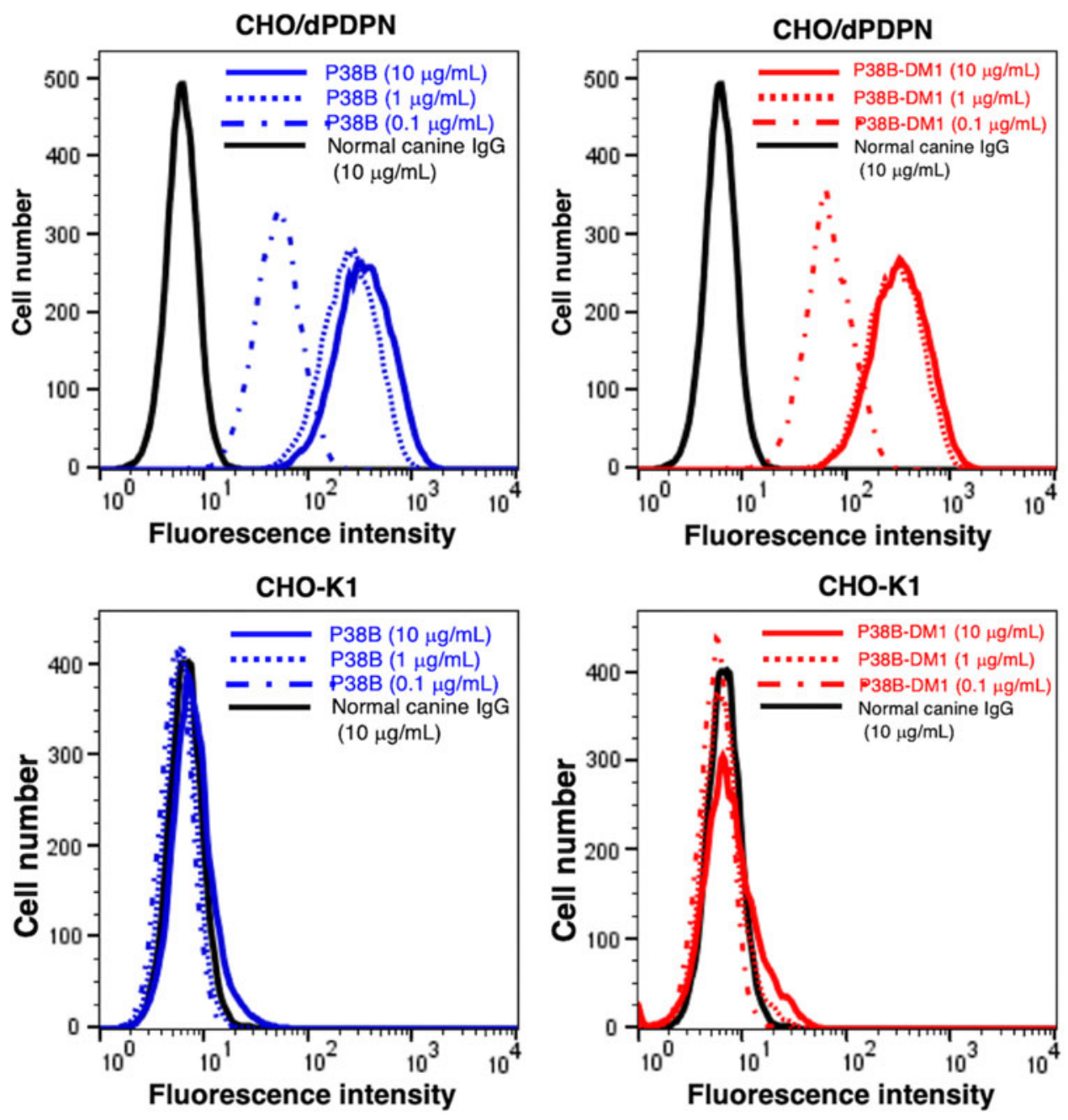

FIG. 2. Flow cytometry. CHO/dPDPN cells were treated with P38B (blue line; 10 or $1 \mu \mathrm{g} / \mathrm{mL}$ ), P38B-DM1 (red line; 10 or $1 \mu \mathrm{g} / \mathrm{mL}$ ), and normal canine IgG (black line; $10 \mu \mathrm{g} / \mathrm{mL}$ ), followed by treatment with fluorescein isothiocyanateconjugated anti-canine IgG. Fluorescence data were collected using a cell analyzer. IgG, immunoglobulin G; CHO, Chinese hamster ovary; PDPN, dog podoplanin. 
manner. Furthermore, P38B-DM1 did not react with CHOK1 (Fig. 2), indicating that it was sensitive and specific against dPDPN.

Next, we investigated the cytotoxicity of P38B and P38BDM1 against CHO/dPDPN cells. P38B-DM1 demonstrated high cytotoxicity in a dose-dependent manner against CHO/dPDPN cells compared with P38B ( $p<0.01$, using Tukey-Kramer test in more than $0.0625 \mu \mathrm{g} / \mathrm{mL})$ and normal canine $\operatorname{IgG}(p<0.01$, using Tukey-Kramer test in more than $0.0625 \mu \mathrm{g} / \mathrm{mL}$ ) (Fig. 3A). In our previous study, $\mathrm{P} 38 \mathrm{~B}$ showed high $\mathrm{ADCC}$ in canine mononuclear cells, and showed CDC using rabbit complement in CHO/dPDPN. ${ }^{(16)}$ In this study, in contrast, the P38B-DM1 showed high cytotoxicity against CHO/dPDPN cells without mononuclear cell complement, indicating that it was internalized into $\mathrm{CHO} / \mathrm{dPDPN}$ cells where DM1 exerted its cytotoxicity. We also performed additional experiments to confirm that P38B$\mathrm{DM} 1$ did not show any toxicity against $\mathrm{CHO}-\mathrm{K} 1$ cells. As given in Figure 3B, P38B-DM1 did not show any toxicity against $\mathrm{CHO}-\mathrm{K} 1$ cells, whereas it killed $\mathrm{CHO} / \mathrm{dPDPN}$ cells.

We further investigated antitumor activities of P38B-DM1 in vivo. $\mathrm{CHO} / \mathrm{dPDPN}$ cells were subcutaneously implanted into the flanks of nude mice. P38B, P38B-DM1, and control (dog $\mathrm{IgG}$ ) were injected three times (on days 1,8 and 14 after cell injection) into the peritoneal cavity of the mice. Tumor formation was clearly observed in mice from the control, P38B-treated, and P38B-DM1-treated groups in CHO/dPDPN xenograft model.

We measured the tumor size in all the xenograft models and found that P38B-DM1 significantly reduced tumor development of the $\mathrm{CHO} / \mathrm{dPDPN}$ xenograft compared with control (dog IgG) on days $8,10,14$, and 17 (Fig. 4). However, the tumor volume in the P38B-DM1-treated groups was not significantly reduced compared with that in the P38B-treated groups because P38B also significantly reduced tumor development compared with control (dog IgG) on all days (Fig. 4). As reported in our previous study, P38B showed very high CDC activity against $\mathrm{CHO} / \mathrm{hPDPN}$ cells, ${ }^{(16)}$ indicating that mouse complement of nude mice might capacitate CDC to P38B.

We next measured the weight of resected tumors from all xenograft models on day 17 . The tumor weight of mice in P38B-DM1-treated groups was significantly lower than that in the control ( $\operatorname{dog} \mathrm{IgG}$ ) group in CHO/dPDPN xenograft models (Fig. 5A). In contrast, the tumor weight of mice in the P38Btreated groups was not significantly reduced compared with the control (Fig. 5A), indicating that P38B-DM1 exerted stronger antitumor activity than P38B. Images of the resected tumors of the $\mathrm{CHO} / \mathrm{dPDPN}$ xenografts are given in Figure $5 \mathrm{~B}$. There were no differences in mouse body weight between the three groups (Fig. 6A), indicating that the P38B-DM1 was not toxic to the mice. The appearance of the mice on day 17 is given in Figure 6B. Because P38B-DM1 could not bind to Fc receptor of mouse mononuclear cells such as NK cells or macrophages, ADCC-mediated killing might not occur in the xenograft models. Although we could not exactly demonstrate the mechanism of anti-tumor activities by P38B-DM1 in this study, P38B-DM1 could be internalized into cells, and exerted cytotoxicity.

\section{Discussion}

Cancer is a deadly disease that affects all eukaryotes. Almost all cancers found in humans are also observed in dogs,
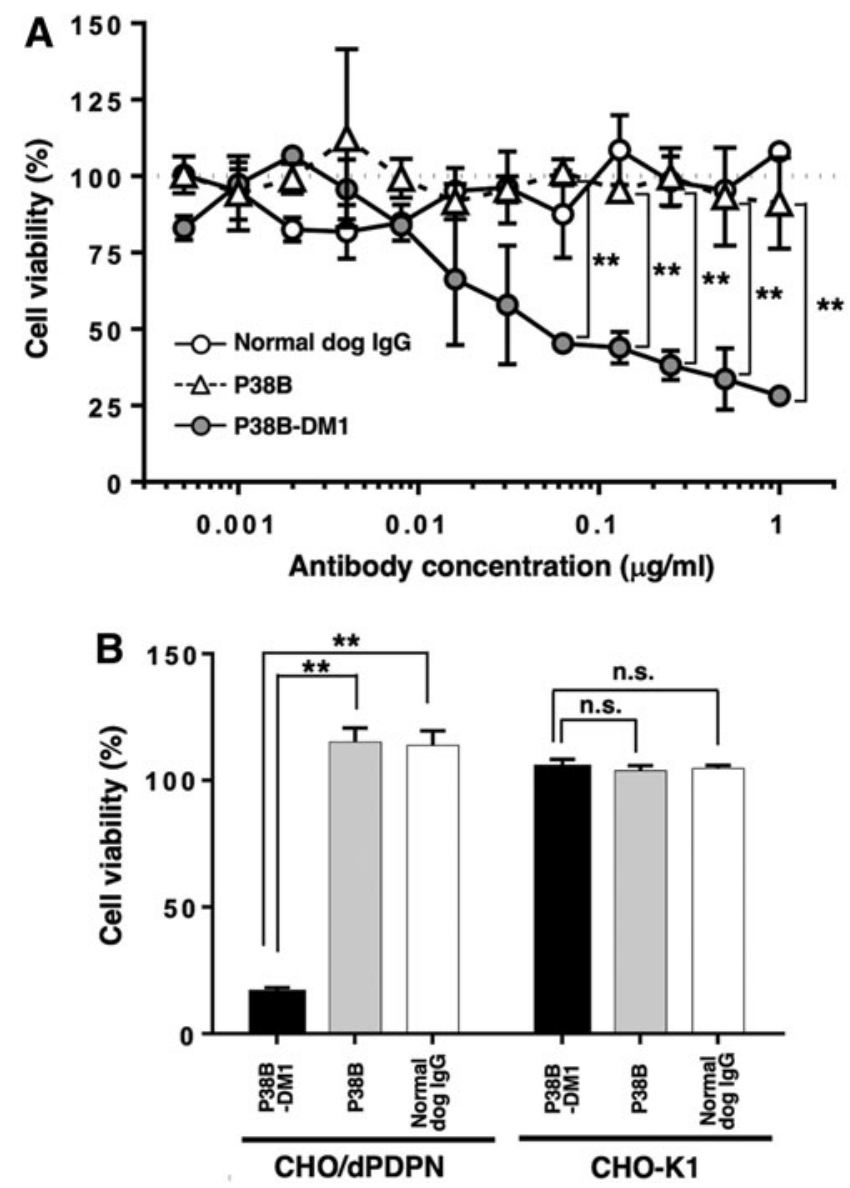

FIG. 3. Cytotoxicity assay. (A) Cytotoxicity assay by cell viability assessment was conducted using CCK-8 We prepared 96-well microplates by plating $\mathrm{CHO} / \mathrm{dPDPN}$ cells at 6000 cells $/ 200 \mu \mathrm{L} /$ well. The plates were incubated for 24 hours at $37^{\circ} \mathrm{C}$ in the presence of $5 \% \mathrm{CO}_{2}$, then serially diluted antibodies were added in a volume of $20 \mu \mathrm{L}$ and cultured for 24 hours. After that, the culture medium was replaced with $100 \mu \mathrm{L}$ of fresh medium with $10 \mu \mathrm{L}$ CCK-8 solution. After a further 5-hour incubation in a $\mathrm{CO}_{2}$ incubator, the absorbance at $450 \mathrm{~nm}$ was measured. The values are presented as mean \pm SEM. Asterisks indicate statistical significance $(* * p<0.01$, Tukey-Kramer test). (B) Cytotoxicity assay by cell viability assessment was conducted using CCK-8 We prepared 96-well microplates by plating $\mathrm{CHO} / \mathrm{dPDPN}$ and $\mathrm{CHO}-\mathrm{K} 1$ cells at 6000 cells $/ 200 \mu \mathrm{L} /$ well. The plates were incubated for 24 hours at $37^{\circ} \mathrm{C}$ in the presence of $5 \% \mathrm{CO}_{2}$, then serially diluted antibodies were added in a volume of $20 \mu \mathrm{L}$ and cultured for 72 hours. After that, the culture medium was replaced with $100 \mu \mathrm{L}$ of fresh medium with $10 \mu \mathrm{L} \mathrm{CCK}-8$ solution. After a further 6-hour incubation in a $\mathrm{CO}_{2}$ incubator, the absorbance at $450 \mathrm{~nm}$ was measured. Values are presented as mean \pm SEM. Asterisks indicate statistical significance $(* * p<0.01$, TukeyKramer test). n.s., not significant; CCK-8, Cell Cloning Kit-8; SEM, standard error of mean.

suggesting that the treatment approaches used in humans may also be effective in canine cancers. Antibody therapies have been shown to improve disease outcome in cancer patients and ADCs are thought to be one of the most potent nextgeneration antibody therapies. ${ }^{(23)}$

We previously produced $\mathrm{P} 38 \mathrm{~B}$, a mouse-canine chimeric anti-dPDPN antibody of canine subclass $\mathrm{B}^{(16)}$ because canine 


\section{CHO/dPDPN}

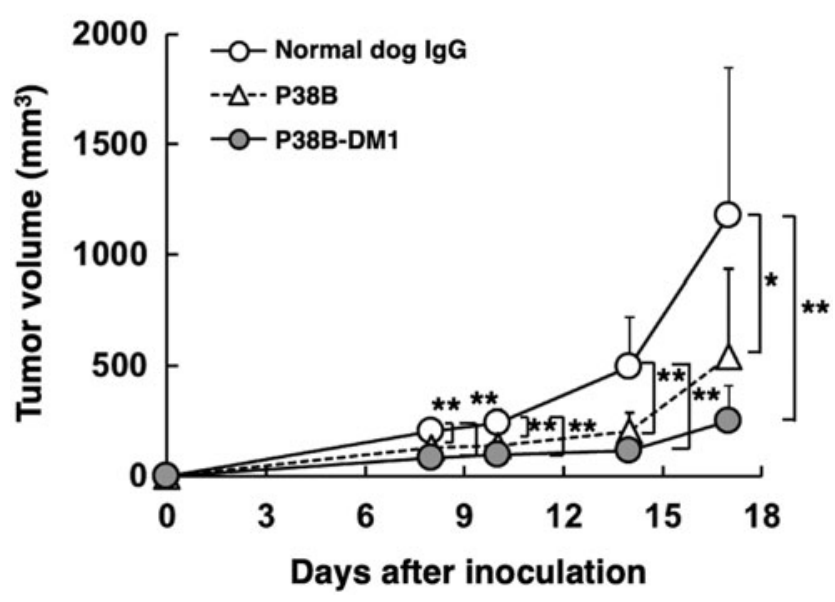

FIG. 4. Antitumor activity of $\mathrm{P} 38 \mathrm{~B}$ and P38B-DM1 against $\mathrm{CHO} / \mathrm{dPDPN}$. Tumor volume of $\mathrm{CHO} / \mathrm{dPDPN}$ xenografts. $\mathrm{CHO} / \mathrm{dPDPN}$ cells were injected subcutaneously into female nude mice. The indicated antibodies $(100 \mu \mathrm{g} /$ day; $5 \mathrm{mg} / \mathrm{kg}$ ) were administered intraperitoneally on days 1,8 , and 14 after cell inoculation. The tumor volume was measured at the indicated time points. The values are presented as mean \pm SEM. Asterisks indicate statistical significance $(* p<0.05, * * p<0.01$, Tukey-Kramer test).

A

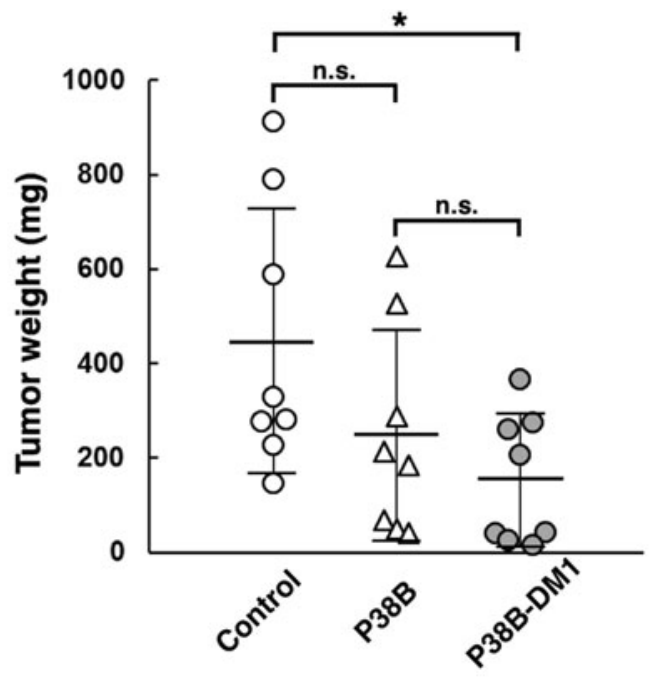

B

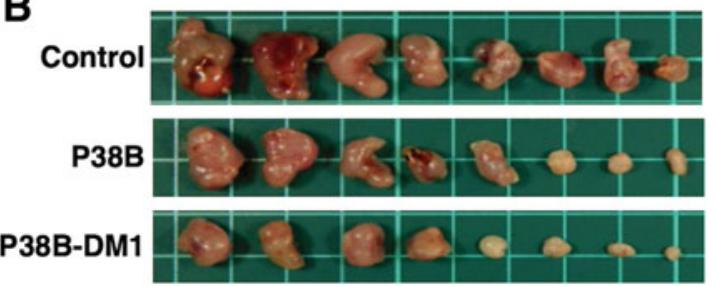

FIG. 5. Evaluation of antitumor activity of $\mathrm{P} 38 \mathrm{~B}$ and P38B-DM1 against CHO/dPDPN. (A) Tumor weight of $\mathrm{CHO/dPDPN}$ xenografts (day 17). (B) Images of resected tumors of CHO/dPDPN xenografts. Values are presented as mean \pm SEM. The asterisk indicates statistical significance $\left({ }^{*} p<0.05\right.$, Tukey-Kramer test). n.s., not significant.

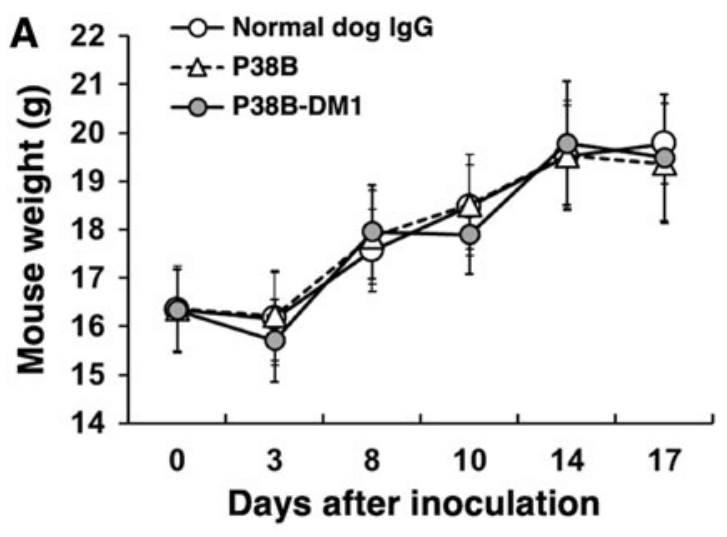

B
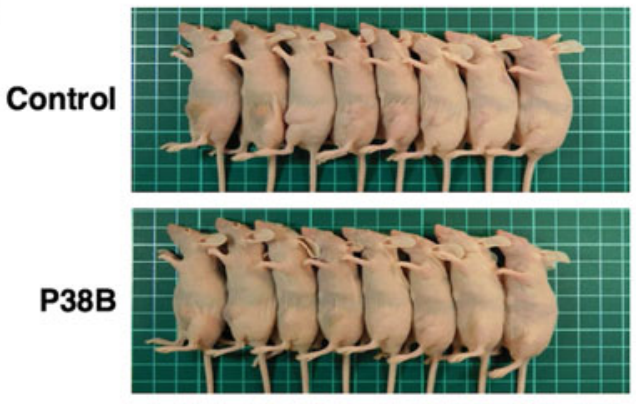

P38B-DM1

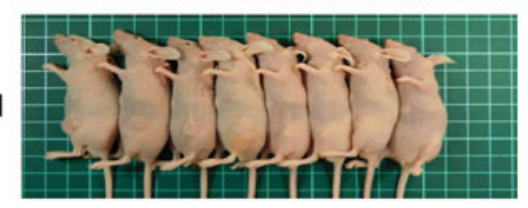

FIG. 6. Evaluation of the safety of $\mathrm{P} 38 \mathrm{~B}$ and $\mathrm{P} 38 \mathrm{~B}-\mathrm{DM} 1$ against $\mathrm{CHO} / \mathrm{dPDPN}$. (A) Body weight of $\mathrm{CHO} / \mathrm{dPDPN}$ xenografts. (B) Images of $\mathrm{CHO} / \mathrm{dPDPN}$ xenograft model mice on day 17.

subclass B possesses ADCC and CDC activities analogous to the human $\mathrm{IgG}_{1}$ subclass. ${ }^{(24)} \mathrm{We}$ clearly demonstrated that $\mathrm{P} 38 \mathrm{~B}$ possesses ADCC and CDC against CHO/dPDPN cells. ${ }^{(16)}$ $\mathrm{P} 38 \mathrm{~B}$ reduced tumor development of $\mathrm{CHO} / \mathrm{dPDPN}$ xenograft significantly compared with control $\operatorname{dog} \operatorname{IgG},{ }^{(17)}$ indicating that P38B exerted antitumor activity against dPDPN-expressing tumors using $\mathrm{ADCC}$ and $\mathrm{CDC}$ activities. In those previous studies, canine mononuclear cells were also injected into xenograft models to induce ADCC activity of P38B. In contrast, antitumor activities of P38B were also observed in this study (Fig. 4), although canine mononuclear cells were not injected into the xenograft models, indicating that $\mathrm{CDC}$ of $\mathrm{P} 38 \mathrm{~B}$ alone could exert antitumor activity. However, the tumor weight of mice in the P38B-treated groups was not reduced significantly compared with the control (dog $\mathrm{IgG})$ group in CHO/dPDPN xenograft models (Fig. 4A), suggesting that CDC of P38B alone did not exhibit sufficient antitumor activity.

In this study, an ADC, P38B-DM1 was generated by conjugating P38B and HSA-attached DM1 (Fig. 1). This ADC showed almost the same binding ability to dPDPN as unmodified P38B (Fig. 2), indicating that the HSA modification of the antibody did not affect antigen binding. Furthermore, its efficacy, as measured by its tumor regression activity, was confirmed by in vitro (Fig. 3) and in vivo (Figs. 4 and 5). P38B + DBCO-HSA-DM-1 conjugates will be intracellularly 
incorporated by endocytosis through the binding with dPDPN. The incorporated conjugate is degraded by proteases in lysosome to generate the DM1-attached Lys amino acid or oligopeptide, which can work as a killing agent through tubulin polymerization inhibition to induce apoptosis. The $\mathrm{IC}_{50}$ of P38B-DM1 was estimated to be $0.048 \mu \mathrm{g} / \mathrm{mL}$ in our preliminary experiment, which corresponds to $238 \mathrm{pM}$, assuming the molecular weight of P38B-DM1 is $\sim 210,000$. This value is larger than the $\mathrm{IC}_{50}$ of T-DM1 (50 pM) against the HER2 + SK-BR3 breast cancer cell line despite the similar DAR for both (2.9 for P38B-DM1 and 3.5 for T-DM1). However, the difference in $\mathrm{IC}_{50}$ may be arise from a difference in the expression levels of the target antigens between the cell types. A detailed comparison in the near future is advised to elucidate the advantages of these ADCs.

In this study, we examined the effects of P38B, a mousecanine chimeric anti-dPDPN antibody of subclass $B$ and its ADC with emtansine (P38B-DM1) on CHO/dPDPN cells. Results indicate a significant increase in the cytotoxicity and antitumor activity of P38B-DM1 against CHO/dPDPN cells in comparison with $\mathrm{P} 38 \mathrm{~B}$ in vitro and in vivo, suggesting that P38B-DM1 is applicable for antibody therapy against canine cancers expressing dPDPN. Although the stability of our ADC has not been checked in serum or in vivo, the toxicity after administration in vivo has not been observed. This suggests that a rapid release of drug (DM1) from ADC did not occur after administration. In principle, we assume the action of mechanism of our ADC, as follows. ADC will be incorporated into the cells by endocytosis through the binding with PDPN. The incorporated ADC will be degraded in late endosome generated with fusion with lysosome into drug (DM1)-attached amino acid (Lys) or oligopeptides, which can work as a killing agent through the inhibition of tubulin polymerization. Further studies on antitumor activities against endogenous dPDPNexpressing tumors are necessary to obtain a more detailed understanding of antibody therapy against canine cancers.

\section{Acknowledgments}

The authors thank Mikiko Yanagawa, Yoshimi Nakamura (Tohoku University); Akiko Harakawa (BIKAKEN), Chisaki Imai, Nana Osako, Daichi Hamada (Kagoshima University) for excellent technical assistance.

\section{Author Disclosure Statement}

No competing financial interests exist.

\section{Funding Information}

This research was supported in part by Japan Agency for Medical Research and Development (AMED) under Grant Nos: JP19am0401013 (Y.K.), $19 \mathrm{am0401002} \mathrm{(Y.I.),}$ JP19am0101078 (Y.K.), and JP19ae0101028 (Y.K.), and by Japan Society for the Promotion of Science (JSPS) KAKENHI (Grant Nos: 17K07299, M.K.K; and 19K07705, Y.K.).

\section{References}

1. Yamada K, Shikida N, Shimbo K, Ito Y, Khedri Z, Matsuda Y, and Mendelsohn BA: AJICAP: Affinity peptide mediated regiodivergent functionalization of native antibodies. Angew Chem Int Ed Engl 2019;58:5592-5597.
2. Yamada $\mathrm{K}$, and Ito $\mathrm{Y}$ : Recent chemical approaches for sitespecific conjugation of native antibodies: Technologies toward next-generation antibody-drug conjugates. Chembiochem 2019;20:2729-2737.

3. Kishimoto S, Nakashimada Y, Yokota R, Hatanaka T, Adachi M, and Ito Y: Site-specific chemical conjugation of antibodies by using affinity peptide for the development of therapeutic antibody format. Bioconjug Chem 2019;30: 698-702.

4. Breiteneder-Geleff S, Matsui K, Soleiman A, Meraner P, Poczewski H, Kalt R, Schaffner G, and Kerjaschki D: Podoplanin, novel 43-kD membrane protein of glomerular epithelial cells, is down-regulated in puromycin nephrosis. Am J Pathol 1997;151:1141-1152.

5. Mishima K, Kato Y, Kaneko MK, Nishikawa R, Hirose T, and Matsutani $\mathrm{M}$ : Increased expression of podoplanin in malignant astrocytic tumors as a novel molecular marker of malignant progression. Acta Neuropathol (Berl) 2006;111: 483-488.

6. Kato Y, Kaneko M, Sata M, Fujita N, Tsuruo T, and Osawa M: Enhanced expression of Aggrus (T1alpha/podoplanin), a platelet-aggregation-inducing factor in lung squamous cell carcinoma. Tumor Biol 2005;26:195-200.

7. Kimura N, and Kimura I: Podoplanin as a marker for mesothelioma. Pathol Int 2005;55:83-86.

8. Abe S, Morita Y, Kaneko MK, Hanibuchi M, Tsujimoto Y, Goto H, Kakiuchi S, Aono Y, Huang J, Sato S, Kishuku M, Taniguchi Y, Azuma M, Kawazoe K, Sekido Y, Yano S, Akiyama S, Sone S, Minakuchi K, Kato Y, and Nishioka Y: A novel targeting therapy of malignant mesothelioma using anti-podoplanin antibody. J Immunol 2013;190:6239-6249.

9. Retzbach EP, Sheehan SA, Nevel EM, Batra A, Phi T, Nguyen ATP, Kato Y, Baredes S, Fatahzadeh M, Shienbaum AJ, and Goldberg GS: Podoplanin emerges as a functionally relevant oral cancer biomarker and therapeutic target. Oral Oncol 2018;78:126-136.

10. Kato Y, and Kaneko MK: A cancer-specific monoclonal antibody recognizes the aberrantly glycosylated podoplanin. Sci Rep 2014;4:5924.

11. Honma R, Kaneko MK, Ogasawara S, Fujii Y, Konnai S, Takagi M, and Kato Y: Specific detection of dog podoplanin expressed in renal glomerulus by a novel monoclonal antibody PMab-38 in immunohistochemistry. Monoclon Antib Immunodiagn Immunother 2016;35:212-216.

12. Zimmer G, Klenk HD, and Herrler G: Identification of a 40$\mathrm{kDa}$ cell surface sialoglycoprotein with the characteristics of a major influenza $\mathrm{C}$ virus receptor in a Madin-Darby canine kidney cell line. J Biol Chem 1995;270:17815-17822.

13. Kaneko MK, Honma R, Ogasawara S, Fujii Y, Nakamura T, Saidoh N, Takagi M, Kagawa Y, Konnai S, and Kato Y: PMab-38 recognizes canine podoplanin of squamous cell carcinomas. Monoclon Antib Immunodiagn Immunother 2016;35:263-266.

14. Ogasawara S, Honma R, Kaneko MK, Fujii Y, Kagawa Y, Konnai S, and Kato Y: Podoplanin expression in canine melanoma. Monoclon Antib Immunodiagn Immunother 2016;35:304-306.

15. Chang YW, Yamada S, Kaneko MK, and Kato Y: Epitope mapping of monoclonal antibody PMab-38 against dog podoplanin. Monoclon Antib Immunodiagn Immunother 2017;36:291-295.

16. Kato Y, Mizuno T, Yamada S, Nakamura T, Itai S, Yanaka M, Sano M, and Kaneko MK: Establishment of P38Bf, a corefucose-deficient mouse-canine chimeric antibody against dog 
podoplanin. Monoclon Antib Immunodiagn Immunother 2018;37:218-223.

17. Kato Y, Ohishi T, Kawada M, Maekawa N, Konnai S, Itai S, Yamada S, and Kaneko MK: The mouse-canine chimeric anti-dog podoplanin antibody P38B exerts antitumor activity in mouse xenograft models. Biochem Biophys Rep 2019;17:23-26.

18. Braisted AC, and Wells JA: Minimizing a binding domain from protein A. Proc Natl Acad Sci U S A 1996;93:56885692.

19. Fujii Y, Kaneko M, Neyazaki M, Nogi T, Kato Y, and Takagi J: PA tag: A versatile protein tagging system using a super high affinity antibody against a dodecapeptide derived from human podoplanin. Protein Expr Purif 2014;95: 240-247.

20. Fujii Y, Kaneko MK, Ogasawara S, Yamada S, Yanaka M, Nakamura T, Saidoh N, Yoshida K, Honma R, and Kato Y: Development of RAP tag, a novel tagging system for protein detection and purification. Monoclon Antib Immunodiagn Immunother 2017;36:68-71.

21. Fujii Y, Kaneko MK, and Kato Y: MAP tag: A novel tagging system for protein purification and detection. Monoclon Antib Immunodiagn Immunother 2016;35:293-299.

22. Kato Y, Kunita A, Abe S, Ogasawara S, Fujii Y, Oki H, Fukayama M, Nishioka Y, and Kaneko MK: The chimeric antibody chLpMab-7 targeting human podoplanin suppresses pulmonary metastasis via $\mathrm{ADCC}$ and $\mathrm{CDC}$ rather than via its neutralizing activity. Oncotarget 2015;6:3600336018.

23. Khongorzul P, Ling CJ, Khan FU, Ihsan AU, and Zhang J: Antibody-drug conjugates: A comprehensive review. Mol Cancer Res 2019;18:3-19.

24. Bergeron LM, McCandless EE, Dunham S, Dunkle B, Zhu Y, Shelly J, Lightle S, Gonzales A, and Bainbridge G: Comparative functional characterization of canine $\mathrm{IgG}$ subclasses. Vet Immunol Immunopathol 2014;157:31-41.

Address correspondence to: Yukinari Kato New Industry Creation Hatchery Center Tohoku University 2-1 Seiryo-machi, Aoba-ku Sendai, Miyagi 980-8575 Japan

E-mail: yukinarikato@med.tohoku.ac.jp

Received: January 13, 2020 Accepted: February 19, 2020 